\title{
New Pulsars from Arecibo Drift-Scan Searches
}

\author{
M. A. McLaughlin, D. R. Lorimer, D. J. Champion \\ University of Manchester, Jodrell Bank Observatory, Macclesfield, UK \\ K. Xilouris \\ University of Virginia, Charlottesville VA, USA
}

Z. Arzoumanian

USRA/LHEA, NASA-GSFC, Greenbelt MA, USA

D. C. Backer

University of California, Berkeley CA, USA

J. M. Cordes

Cornell University, Ithaca NY, USA

A. S. Fruchter

Space Telescope Science Institute, Baltimore MD, USA

\author{
A. N. Lommen \\ Franklin \& Marshall College, Lancaster PA, USA
}

\begin{abstract}
We report on new pulsars discovered in Arecibo drift-scan data. Processing of $2200 \mathrm{deg}^{2}$ of data has resulted in the detection of 41 known and 12 new pulsars. New pulsars include two millisecond pulsars, one solitary and one binary recycled pulsar, and one pulsar with very unusual pulse profile morphology and complex drifting subpulse behavior.
\end{abstract}

\section{Results}

In McLaughlin et al. (2003) we describe the data collection and analysis procedures for this survey. At the completion of this analysis, we have redetected 41 known pulsars and 12 new pulsars. These new pulsars are J0152+09 $(P$ $=915 \mathrm{~ms}), \mathrm{J} 0546+24(P=2.8 \mathrm{~s}), \mathrm{J} 0609+21 \quad(P=56 \mathrm{~ms}), \mathrm{J} 0815+09(P=$ $845 \mathrm{~ms}), \mathrm{J} 1453+19(P=5.8 \mathrm{~ms}), \mathrm{J} 1504+21(P=3.3 \mathrm{~s}), \mathrm{J} 1746+22(P=3.5 \mathrm{~s})$, $\mathrm{J} 1823+06(P=753 \mathrm{~ms}), \mathrm{J} 1829+24(P=41 \mathrm{~ms}), \mathrm{J} 1944+09(P=5.2 \mathrm{~ms})$, $\mathrm{J} 2007+09(P=458 \mathrm{~ms}) \& \mathrm{~J} 2045+09(P=395 \mathrm{~ms})$.

PSR J0609+2130: From a year of timing, we establish that this pulsar is recycled and is a solitary neutron star (Lorimer et al. 2004). It and PSR J2235+1506 (Camilo et al. 1993) both lie in a part of the $P-\dot{P}$ diagram consisting almost 

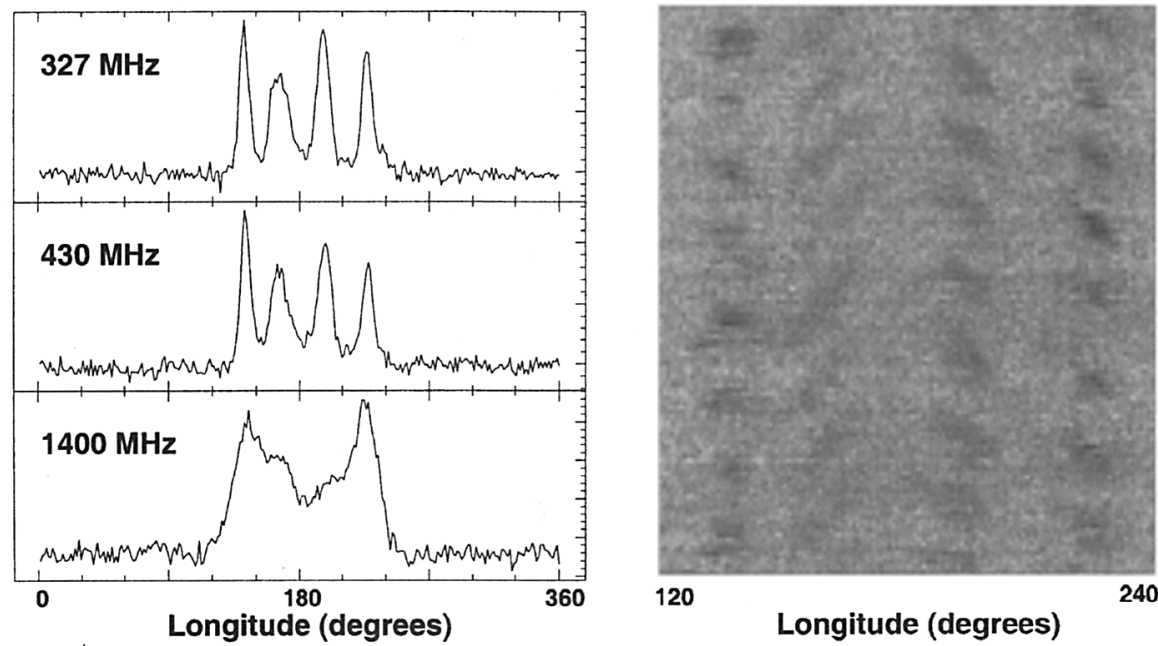

Figure 1. Left: Multi-frequency pulse profiles of J0815+09. Right: Sequence of individual pulses at $327 \mathrm{MHz}$.

completely of binary pulsars. We hypothesize that these unique objects are the end-products of massive binary systems that disrupted following the masstransfer phase due to the supernova explosion of the companion star. We should be able to measure a proper motion within 1-2 years, offering us insights into the formation of these isolated pulsars.

PSR J0815+09: As shown in Figure 1, the profile morphology is not easily explained by standard models. In addition, drifting is seen in all components with $P_{3}=15 P_{1}$ and $P_{2}=0.05 P_{1}$; the sense of drift differs in different components, with no sense reversals. These properties are consistent at multiple epochs and frequencies. No timing irregularities are apparent; the derived age and magnetic field are $10 \mathrm{Myr}$ and $8.1 \times 10^{11} \mathrm{G}$, respectively.

PSR J1829+24: Timing observations of this pulsar over a 4-month timespan reveal a binary orbit of 1.2 days, with eccentricity of 0.14 and projected semimajor axis of 7.2 light seconds. The minimum companion mass is $1.28 M_{\odot}$, making the companion most likely a neutron star. We derive a coalescence time of $\sim 50$ Gyr. This pulsar is quite similar to PSR J1518+4904 (Nice et al. 1996), but has a more compact orbit and hence an expected $\dot{\omega}$ which is $\sim 30$ times larger.

\section{References}

Camilo, F., Nice, D. J., \& Taylor, J. H. 1993, ApJ, 412, L37

Lorimer D. R. et al. 2004, MNRAS, 347, L21

McLaughlin, M. A. et al. 2003, in ASP Conf. Series, Vol. 302, Radio Pulsars, eds. M. Bailes, D. J. Nice, \& S. E. Thorsett, (San Francisco: ASP), p. 129

Nice, D. J., Sayer, R. W., \& Taylor, J. H. 1996, ApJ, 466, 87 